\title{
Performance of Frequency Domain DFE Using
}

\section{Subcarrier Allocation}

\author{
${ }^{1,2}$ P.SIVAKUMAR, ${ }^{3}$ Dr.M.RAJARAM \\ ${ }^{1}$ Research Scholar, ANNA University Chennai INDIA \\ ${ }^{2}$ Assistant Professor /ECE SKP Engineering College Tiruvannamalai, INDIA \\ ${ }^{3}$ Vice Chancellor ANNA University of Technology Tirunelveli, INDIA
}

\begin{abstract}
Due to the usage of single carrier, the performance of SC-FDMA systems degrades in deep frequency selective fading channels. In this paper, we propose a structure of equalizer based on frequency domain decision feedback which could be used for multi-user SC-FDMA systems. This algorithm is applicable to various carrier allocations in multi-user systems such as localized allocation, distributed allocation, and frequency-hopping (FH) allocation. To reduce the complexity, it is not necessary to derive the inversion of matrix, which is required in the traditional decision feedback equalizer for single carrier frequency domain equalization (SC-FDE-DFE). Simulation results show that equalization has been achieved for FD-LE and FDDFE with distribution mapping. This structure can be used in the broadcasting uplink channels with SC-FDMA scheme.
\end{abstract}

Index Terms-Decision feedback equalizer (DFE), frequency domain, SC-FDMA, uplink.

\section{Introduction}

DIGITAL terrestrial television broadcasting (DTTB) has been a very popular broadcasting transmission system in the world. Recently, the time domain synchronous orthogonal frequency division multiplex (TDS-OFDM) technique was adopted as the Chinese national DTTB standard, named as Digital terrestrial Television
Multimedia Broadcasting (DTMB) system [1]-[3]. Instead of using cyclic-prefix (CP), TDS-OFDM uses a short pseudo-random noise (PN) sequence as the guard interval, which can also be used for synchronization and channel estimation [4], [5]. As an important addition of the DTTB system, interactive uplink channel can provide more user oriented services and expanded operations. The European Telecommunications Standards Institute (ETSI) has launched the Digital Video Broadcasting - Return Channel through Terrestrial (DVB-RCT) [6], [7] as the uplink channel solution, which uses the Orthogonal Frequency Division Multiple Access (OFDMA) as the multiple access technique.

In addition, Single-Carrier Frequency Division Multiple Access (SC-FDMA) has been extensively adopted as a typical uplink transmission method for multiuser access scenarios. Compared with OFDMA scheme, SC-FDMA system has its own advantages, such as low Peak to Average Power Ratio (PAPR).Low PAPR can allow the system to relax the specifications of linearity in power amplifier of the mobile terminal, which will reduce the cost and the power consumption. Since SC-FDMA is an effective method used in wireless broadband communication system, it has been chosen as the uplink transmission method in Long Term Evolution (LTE) system [8], which supports $1.25-20 \mathrm{MHz}$ bandwidth and 
high uplink transmission rate up to $50 \mathrm{Mbps}$. Therefore, SC-FDMA scheme is more suitable for uplink channel due to its lower power consumption

The frequency domain equalization (FDE) with the use of a single carrier (SC) scheme, referred to as SC-FDE, has been considered as a low-complexity scheme for broadband wireless These systems are known to be simply derived from multicarrier (MC) transmission systems, such as orthogonal frequency-division multiplexing (OFDM), by shifting the inverse discrete Fourier transform (IDFT) from transmitter to the receiver end.

In the SC-FDMA system, the first-order frequency domain linear equalizer (FD-LE) could be adopted to perform the equalization after discrete Fourier transform (DFT) which transforms the received signal from time domain to frequency domain. However, in deep frequency selective fading channels spectral nulls of channel frequency response (CFR) appeal in some specific frequencies. Since the effect of every single frequency point can cause damages to all the time domain constellation symbols, the noise signal in spectral nulls are significantly amplified when FD-LE is used. Therefore the performance of FD-LE deteriorates in high frequency selective and deep fading channels. Decision feedback is a common technique employed in single carrier system to optimize the performance under such kind of conditions. In [9], [10], hybrid-domain equalization architecture is proposed for single carrier systems which is known as decision feedback equalizer for single carrier frequency domain equalization (SC-FDE-DFE), in which FD-LE is conducted as forward equalizer and time domain transverse filter is adopted as feedback equalization. Based on the same principle, the structure of time domain noise predication decision feedback equalization is considered as the deformation of hybrid-domain equalizer in [11]. [12] Introduces feedback filter and iterative design method into frequency domain. In [13] the traditional SCFDE-DFE is applied to SC-FDMA system for each single user.
In this paper, we design novel frequency domain decision feedback equalization (DFE) structure that has the united form for all three kinds of subcarrier distributions namely localized allocation, distributed allocation and frequency hopping (FH) allocation for multi-user SC-FDMA system in the first time. This strategy reduces the complexity by replacing the operation to get the inversion of matrix in SC-FDE-DFE with simple DFT transform. Simulations in multi-user uplink systems illustrate that the performance of this approach is effective under various resource distribution methods.

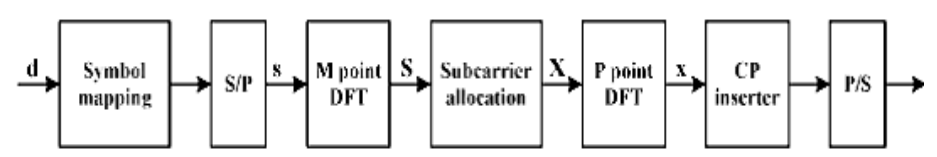

(a)

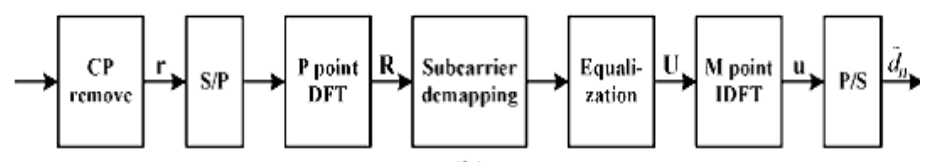

(b)

Fig. 1. Block diagram of SC-FDMA system

This paper is organized as follows. In Section II, both transmitter and receiver of SC-FDMA systems are described and analyzed according to three different resource allocation methods. The multi-user frequency domain DFE is proposed in Section III, and the parameters are defined based on minimum mean square error (MMSE) rule in both ideal feedback and actual case when decision feedback errors are considered. And in Section IV simulation results are presented to verify the benefits of the proposed algorithm. Finally, conclusions are drawn in Section V.

\section{System Description}

In this section, both transmitters of the SC-FDMA systems are described. Three different sub-carrier allocation schemes, i.e. localized, distributed and frequency hopping allocations are analyzed. 
It is assumed that the total number of users in SCFDMA systems is $\mathrm{K}$. The diagram of the transmitter for each user is shown in Fig. (1). The number of subcarriers for each user is $M$. The total sub-carriers used in the system is $\mathrm{P}$. so we can get $\mathrm{P}=\mathrm{KM}$. For the Kth user, the input bit stream is converted to constellation symbols then grouped into a data block $\mathrm{S}^{(\mathrm{k})}$ of size M,i.e $\mathrm{s}^{(\mathrm{k})}$ $=\left[\mathrm{S}_{0}{ }^{(\mathrm{k})}, \mathrm{S}_{1}{ }^{(\mathrm{k})}, \ldots . \mathrm{S}_{\mathrm{M}-1}{ }^{(\mathrm{k})}\right]^{\mathrm{T}}$. After that, $\mathrm{S}^{(\mathrm{k})}$ transformed to frequency domain by a M-point DFT. According to different resource allocation schemes, is mapped to subcarriers which can be expressed as

$$
\mathbf{X}^{(k)}=\mathbf{D}^{(k)} \mathbf{S}^{(k)} \quad k=1,2, \ldots, K
$$

Where $\mathrm{D}^{(\mathrm{k})}$ is the resource allocation matrix with size of $\mathrm{P} \times \mathrm{M}$. For different schemes, is expressed as follows

$$
\begin{aligned}
& \mathbf{D}_{L o c,(m, n)}^{(k)}= \begin{cases}1, & m=(k-1) M+n, \quad 0 \leq n \leq M-1 \\
0, & \text { otherwise }\end{cases}
\end{aligned}
$$

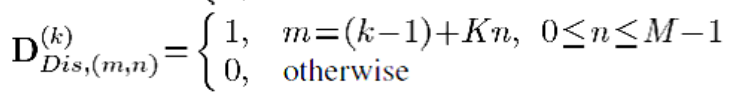

$$
\begin{aligned}
& \mathbf{D}_{F H,(m, n)}^{(k)}= \begin{cases}1, & m=\mathrm{FH}^{(k)}(n), \quad 0 \leq n \leq M-1 \\
0, & \text { otherwise }\end{cases}
\end{aligned}
$$

where , $\mathrm{D}_{\mathrm{Loc}}{ }^{(\mathrm{k})} \mathrm{D}_{\mathrm{Dis}}{ }^{(\mathrm{k})}$ and $\mathrm{D}_{\mathrm{FH}}{ }^{(\mathrm{k})}$ denote resource allocation matrixes of localized, distributed and frequency hopping methods of the Kth user respectively, $(m, n)$ is the element index, $0 \leq \mathrm{m} \leq \mathrm{P}-1,0 \leq \mathrm{n} \leq \mathrm{M}-1 . \mathrm{FH}^{(\mathrm{k})}(\mathrm{n})$ represents the frequency hopping function of the K th user. From (2)-(4), a general expression of the mapping process is obtained as

$$
\mathbf{D}_{(m, n)}^{(k)}= \begin{cases}1, & m=\mathrm{MP}^{(k)}(n), \quad 0 \leq n \leq M-1 \\ 0, & \text { otherwise }\end{cases}
$$

Where $\mathrm{D}_{(\mathrm{m}, \mathrm{n})}{ }^{(\mathrm{k})}$ is the $(\mathrm{m}, \mathrm{n})$ element of $\mathrm{D}^{(\mathrm{k})}, \mathrm{MP}^{(\mathrm{k})}(\mathrm{n})$ is the subcarrier allocation function of the Kth user. For different users ${ }^{(\mathrm{k})}$ are orthogonal.

$$
\mathbf{D}^{\left(k_{1}\right) \mathrm{T}} \mathbf{D}^{\left(k_{2}\right)}= \begin{cases}\mathbf{I}_{M}, & k_{1}=k_{2} \\ \mathbf{O}_{M}, & k_{1} \neq k_{2}\end{cases}
$$

Where the superscript $\mathrm{T}$ denotes the matrix transpose, $\mathrm{I}_{\mathrm{M}}$ is an identify matrix with size $\mathrm{MX} \mathrm{M}, \mathrm{O}_{\mathrm{M}}$ is a $\mathrm{MxM}$ size all-zero matrix.

After the sub-carrier mapping, time domain data block is obtained by a -point inverse DFT (IDFT). The SCFDMA signal is generated after inserting $\mathrm{CP}$ and converting to serial. The transmitted signal is shown as follows

$$
\mathbf{x}^{(k)}=\mathbf{W}_{P}^{\mathrm{H}} \mathbf{D}^{(k)} \mathbf{W}_{M} \mathbf{s}^{(k)}
$$

Where $\mathrm{H}$ denotes the hermitian matrix, $\mathrm{W}_{\mathrm{p}}$ is a $\mathrm{P} \mathrm{X} \mathrm{P}$ DFT matrix, i.e. $\mathrm{W}_{\mathrm{p}}(\mathrm{m}, \mathrm{n})=\mathrm{e}^{-\mathrm{j} 2 \pi / \mathrm{P} \cdot \mathrm{mn}}, \mathrm{m}, \mathrm{n}=0,1, \ldots, \mathrm{P}-1 \mathrm{~W}_{\mathrm{M}}$ is an M X M DFT matrix. Assuming the channel impulse response for Kth user is, $h^{(k)}$ which can be modeled as $h^{(k)}$ $=\left[\mathrm{h}_{0}{ }^{(\mathrm{k})}, \mathrm{h}_{1}{ }^{(\mathrm{k})} \ldots \mathrm{h}_{\mathrm{Lk}}{ }^{(\mathrm{k})}\right]^{\mathrm{T}}$. The maximum delay spread is which is assumed to be shorter than the length of CP to eliminate the inter-symbol interference (ISI).

\section{Frequency Domain DFE}

When the channel is in deep frequency selective fading, the noise at the null point of the CFR will be amplified significantly. So the enlarged noise could have effect on all the time domain constellation symbols and the performance of the SC-FDMA
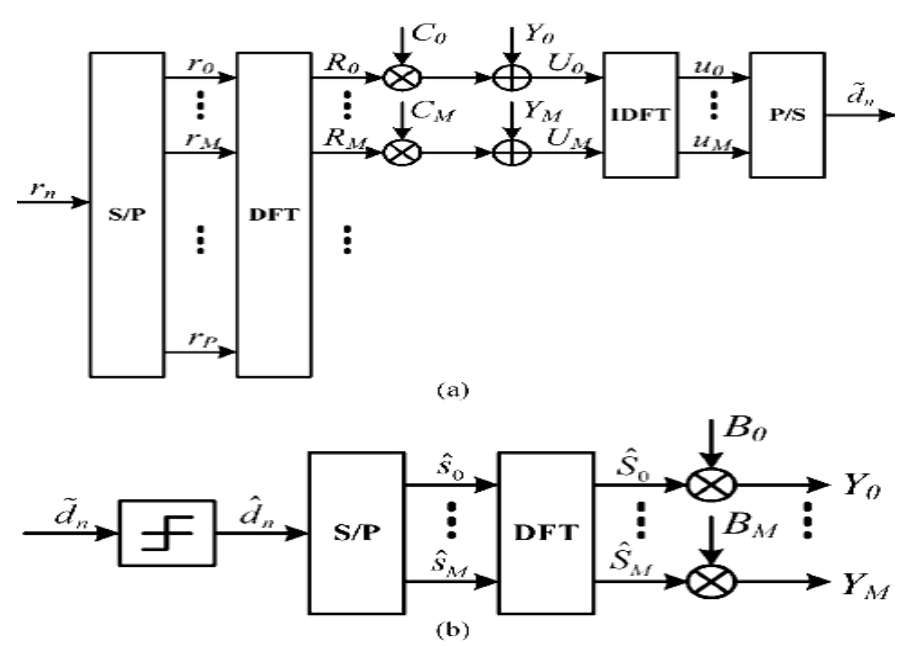

(b)

Fig. 2. Structure of FD-DFE 
System will degrade. Decision feedback is an effective method to deal with deep frequency selective fading for single carrier systems, and can be extended here into SCFDMA

In this section, the proposed frequency domain decision feedback equalization (FD-DFE) suitable for SCFDMA is analyzed. The structure of FD-DFE is shown in Fig. 2. Fig. (2a) describes the forward filter part and Fig. (2b) illustrates the feedback part.

A. ideal Feedback

As shown in Fig.2. For Kth user, the output of the equalizer can be expressed as

$$
u_{m}^{(k)}=\frac{1}{M} \sum_{l=0}^{M-1}\left(C_{l}^{(k)} R_{l}^{(k)}+B_{l}^{(k)} S_{l}^{(k)}\right) e^{j \frac{2 \pi}{M} l m}
$$

After the decision the mean square error (MSE) is

$$
M S E=E\left(u_{m}^{(\boldsymbol{k})}-s_{m}^{(k)^{2}}\right)
$$

With

$S_{l}^{(k)}=\sum_{i=0}^{M-1} s_{i}^{(k)} e^{-j(2 \pi / M) i l} \quad 0 \leq l \leq M-1 .$. we put equation (11) into (12) and obtain

$$
M S E=\left.\frac{P \sigma_{V}^{2}}{M^{2}} \sum_{l=0}^{M-1} C_{l}^{(k)}\right|^{2}+\frac{\sigma_{s}^{2}}{M} \sum_{l=0}^{M-1} \mid C_{l}^{(k)} \tilde{H}_{l}^{(k)}+B_{l}^{(k)}{ }^{2}
$$

With the constraint of $\sum_{l=0}^{M-1} B_{l}^{(k)}=0[12]$, we use Lagrange multiplier method to solve the minimum value of MSE. Construct the objective function $f\left(\mathbf{B}^{(k)}, \mathbf{C}^{(k)}, \lambda\right)$.

$$
\begin{aligned}
& f\left(\mathbf{B}^{(k)}, \mathbf{C}^{(\alpha)}, \lambda\right)=\frac{P \sigma_{l}^{2}}{M^{2}} \sum_{l=0}^{M-1} C_{l}^{(\alpha)}{ }^{2} \\
& +\frac{\sigma_{s}^{2}}{M} \sum_{l=0}^{M-1} C_{l}^{(k)} \dot{H}_{l}^{(d)}+B_{l}^{(d)}{ }^{2}+\lambda \cdot \sum_{l=0}^{M-1} B_{l}^{(d)}
\end{aligned}
$$

By setting the gradient of(14) with respect to $\mathrm{B}_{1}^{(\mathrm{K})}$, $\mathrm{C}_{\mathrm{l}}^{(\mathrm{K})}$ and $\lambda$ to zero, we obtain

$$
\begin{aligned}
\frac{\partial f\left(\mathbf{B}^{(k)}, \mathbf{C}^{(k)}, \lambda\right)}{\partial C_{l}^{(k)}}= & \frac{P \sigma_{V}^{2}}{M^{2}} C_{l}^{(k)}+\frac{\sigma_{s}^{2}}{M} \\
& \times\left(C_{l}^{(k)} \dot{H}_{l}^{(k)}+B_{l}^{(k)}-1\right) \ddot{H}_{l}^{(k) *}=0 \\
\frac{\partial f\left(\mathbf{B}^{(k)}, \mathbf{C}^{(k)}, \lambda\right)}{\partial B_{l}^{(k)}}= & \frac{\sigma_{s}^{2}}{M}\left(C_{l}^{(k)} \ddot{H}_{l}^{(k)}+B_{l}^{(k)}-1\right)+\lambda=0 \\
\frac{\partial f\left(\mathbf{B}^{(k)}, \mathbf{C}^{(k)}, \lambda\right)}{\partial \lambda}= & \sum_{i=0}^{M-1} B_{i}^{(k)}=0
\end{aligned}
$$

From (16) and (17) we can get

$$
\begin{aligned}
B_{l}^{(k)} & =-\left(C_{l}^{(k)} \ddot{H}_{l}^{(k)}-\frac{1}{M} \sum_{i=0}^{M-1} C_{i}^{(k)} \ddot{H}_{i}^{(k)}\right) \\
& =-\left(C_{l}^{(k)} \ddot{H}_{l}^{(k)}-\beta^{(k)}\right)
\end{aligned}
$$

Where

$$
\beta^{(k)}=\frac{1}{M} \sum_{i=0}^{M-1} C_{i}^{(k)} \ddot{H}_{i}^{(k)}
$$

By introducing (18) into (14), the parameter $\mathrm{C}_{\mathrm{l}}^{(\mathrm{K})}$ and $\beta_{1}{ }^{(\mathrm{K})}$ By solving the equations,

$$
\begin{aligned}
C_{l}^{(k)} & =\frac{M \sigma_{z}^{2} n H_{l}^{(k) *}}{P \sigma_{V}^{2}+\sigma_{s}^{2} \sum_{i=0}^{M-1} \ddot{H}_{i}^{(k)^{2}}} \\
\beta^{(k)} & =\frac{\sigma_{s}^{2} \sum_{l=0}^{M-1}\left|\ddot{H}_{l}^{(k)}\right|^{2}}{P \sigma_{V}^{2}+\sigma_{s}^{2} \sum_{l=0}^{M-1} \ddot{H}_{l}^{(k)}{ }^{2}}
\end{aligned}
$$

And MSE is

$$
M M S E=\frac{\left.\sigma_{s}^{4} \sum_{l=0}^{M-1} \ddot{H}_{l}^{(k)}\right|^{2}}{P \sigma_{V}^{2}+\sigma_{s}^{2} \sum_{l=0}^{M-1}\left|\dot{H}_{l}^{(k)}\right|^{2}}
$$




\section{Simulation Results}

Based on the above analyses, simulations are performed to evaluate the performance of the proposed frequency domain decision feedback equalization algorithms in multi-user SC-FDMA systems.

The number of subcarriers is $\mathrm{p}=1024$ and the length of $\mathrm{CP}$ is $\mathrm{p} / 8=128$. The number of users in this system is $\mathrm{K}=8$, then $\mathrm{M}=128$ subcarriers can be allocated to each user. Quaternary Phase Shift Key (QPSK) constellation is selected. Since the channel conditions forever user are varying, 6-path channel model is chosen where the delay of the 6 paths distributes uniformly between 0 to 127 taps and the path power spectrum has exponential distribution. During the simulations, channel estimation and synchronization are assumed ideal.

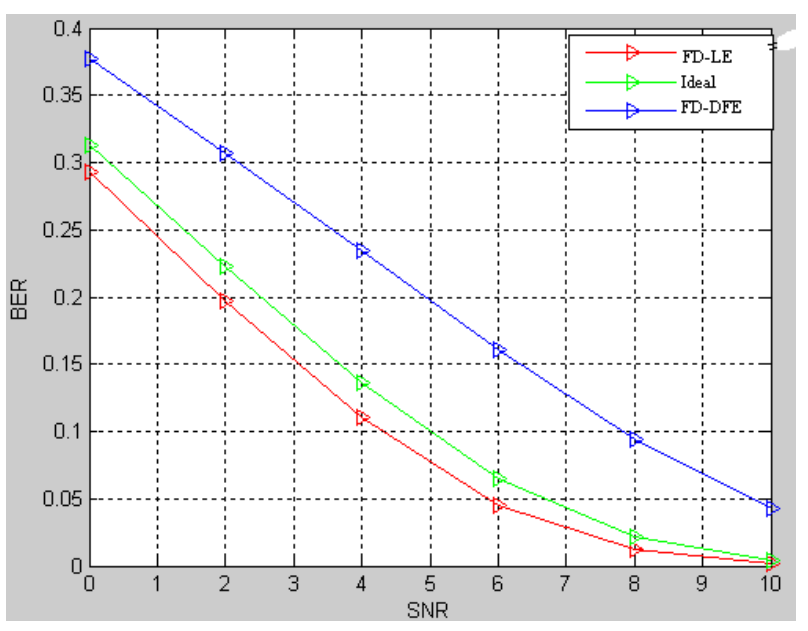

Fig 3. BER Performance of coded system

Fig.3 shows BER performances of the coded SCFDMA system under various equalization schemes. A 1/2-rate convolutional encode $(133,171)$ is used at the transmitter and a soft-decision Viterbi decoder is used in the receiver. The same curves are provided in this figure. For the three sub-carrier allocation methods, the performance of the non-ideal FD-DFE is around $2 \mathrm{~dB}$ better than FD-LE at BER $=1.0 \mathrm{e}-5$. For localized and distributed allocation, the performance difference between non-ideal FD-DFE and ideal FD-DFE is about $2 \mathrm{~dB}$ at $\mathrm{BER}=1.0 \mathrm{e}-5$. In the $\mathrm{FH}$ allocation, the loss due to the decision errors is about $1 \mathrm{~dB}$. For non-ideal feedback, the performance of $\mathrm{FH}$ allocation is about $1 \mathrm{~dB}$ better than that of the others.

\section{Conclusions}

In this paper, a frequency domain decision feedback equalizer suitable for multi-user SC-FDMA systems has been proposed. Based on the MMSE criterion, the forward and feedback filter coefficients are derived theoretically. The proposed FD-DFE can be used for the three subcarrier allocation methods with the same DFE structure and parameter calculation method. Compared with traditional SC-FDE-DFE, since there is no matrix inversion and solving equations in this scheme, the computation complexity is low. Simulations show that the proposed FD-DFE can improve the performance of the multi-user SC-FDMA system significantly when channel is in deep frequency selective fading. This FD-DFE can be used in the broadcasting uplink channel combined with SC-FDMA scheme.

\section{References}

[1] Framing Structure, Channel Coding and Modulation for Digital Television Terrestrial Broadcasting System, P.R. China Standard GB20600-2006, Aug. 18, 2006.

[2] J. Song, Z. X. Yang, L. Yang, K. Gong, C. Y. Pan, J. Wang, and Y. S.Wu, "Technical review on Chinese digital terrestrial television broadcasting standard and measurements on some working modes," IEEE Trans. Broadcasting, vol. 53, no. 1, pp. 1-7, Mar. 2007.

[3] W. J. Zhang, Y. F. Guan, W. Q. Liang, D. Z. He, F. Ju, and J. Sun, "An introduction of the Chinese DTTB standard and analysis of the PN595working modes," IEEE Trans. Broadcasting, vol. 53, no. 1, pp. 8-13,Mar. 2007.

[4] J. Fu, C. Y. Pan, Z. X. Yang, and L. Yang, "Low-complexity equalization for TDS-OFDM systems over doubly selective channels," IEEETrans. Broadcasting, vol. 51, no. 3, pp. 401-407, Sep. 2005.

[5] Z. X. Yang, J. Wang, C. Y. Pan, L. Yang, and Z. Han, "Channel estimation of DMB-T," in 2002 IEEE Int. Conf. Communications, Circuits and Systems and West Sino Expositions, 2002, vol. 2, pp. 1069-1072. 
[6] European Telecommunications Standards Institute (ETSI), "Digital video broadcasting (DVB); Interaction channel for Digital Terrestrial Television (RCT) Incorporating Multiple Access OFDM", EN 301958, v1.1.1, ETSI, Mar. 2002.

[7] D. Prendergast, B. Caron, and Y.Wu, "The implementation of a return channel for ATSC-DTV," IEEE Trans. Broadcasting, vol. 53, no. 2, pp.521-529, Jun. 2007.

[8] 3GPP, "Physical Channels and Modulation," TS 36.211 v8.5.0, 2008.

[9] D. Falconer, S. L. Ariyavisitakul, A. Benyamin-Seeyar, and B. Eidson,"Frequency domain equalization for single carrier broadband wireless systems," IEEE Commun. Mag., no. 4, pp. 58-66, Apr. 2002.

[10] H. Witschnig, M. Kemptner, R. Weigel, and A. Springer, "Decision feedback equalization for single carrier system with frequency domain equalization-An overall system approach," in 2004 1st International Symposium on Wireless Communication Systems, Sep. 2004, pp.26-30.

[11] Y. Zhu and K. B. Letaief, "Single carrier frequency domain equalization with noise prediction for broadband wireless systems," in GLOBECOM' 04, IEEE, Nov. 2004, vol. 5, pp. 3098-3102.

[12] N. Benvenuto and S. Tomasin, "Iterative design and detection of a DFE in frequency domain," IEEE Trans. Commun., vol. 53, no. 11, pp.1867-1875, Nov. 2005.

[13] G. Huang, A. Nix, and S. Armour, "Decision feedback equalization in SC-FDMA," in 2008 19th International Symposium on Personal, Indoor and Mobile Radio Communications, Sep. 2008, pp. 1-5.

\section{AUTHOR PROFILE}

P.Sivakumar has obtained B.E., (ECE) Degree from University of MADRAS in 1999 and completed his M.Tech (DEAC) from National Institute of Technology Karnataka Surathkal (NITK) in 2005. He is a Ph.D research scholar of Anna University; Chennai. He is presently working as Assistant Professor in ECE department of S.K.P. Engineering College, Thiruvannamalai, India. He is a member of ISTE, IETE and international association of engineers (IAENG) Hong Kong. His areas of interest are wireless communication, signal processing and image processing.
Dr.M.Rajaram is vice chancellor of Anna University of technology Thirunelveli India. Previously he worked as Professor and Head of the Department of EEE in GCE, Thirunelveli. He has more than twenty five years of teaching and research experience. His research focuses on network security, image and video processing, FPGA architectures and power electronics. 